\title{
Two-year follow-up after endobronchial coil treatment in emphysema: results from the REVOLENS study
}

\author{
To the Editor:
}

Severe emphysema is a difficult to treat condition with limited efficacy of currently available treatments. Endobronchial coil treatment (ECT) is a minimally invasive endobronchial treatment which consists of placing shape-memory nitinol coils in emphysematous lobes to enhance lung recoil and reduce lung hyperinflation at rest and during exercise $[1,2]$. Randomised studies demonstrated an improvement in exercise capacity, lung function and quality of life, and showed an acceptable safety profile at 1 year [3-6]. However, to our best knowledge, longer term safety and effectiveness results beyond 1 year have not been reported thus far.

REVOLENS (Réduction volumique endobronchique par spirales) (NCT01822795) is a 1:1 randomised controlled study of 100 patients (50 patients treated with coils and optimal medical management and 50 patients treated with optimal medical management only) in 10 centres across France. Patients treated with optimal medical management only were offered coil treatment after 1 year. The study protocol [7] and 1 -year results [5] of the REVOLENS study have been previously described, demonstrating a decrease in lung hyperinflation and improvement in quality of life. In the present study, we analysed efficacy and safety data at 2 years in the 50 patients randomised to the ECT group. The ethics committee of Dijon Est I and the French Agency for Medicines and Health Products Health approved the study protocol.

Among 50 patients treated by ECT, vital status at 2 years was determined for 49 patients. Four patients died within the first year (one each from peritonitis, mesenteric ischaemia, and chronic obstructive pulmonary disease (COPD) exacerbation; one cause was undetermined) and two patients died within the second year (one from COPD exacerbation and the other from undetermined causes). One patient underwent lung transplantation within the second year. 32 patients attended the 2 -year follow-up visit and 11 patients did not. Patients who died or underwent lung transplantation within 2 years $(n=7)$ had lower body mass index $\left(23 \pm 4\right.$ versus $\left.19 \pm 3 \mathrm{~kg} \cdot \mathrm{m}^{-2}, \mathrm{p}=0.03\right)$ and forced expiratory volume in $1 \mathrm{~s}(\mathrm{FEV} 1)(27 \pm 7$ versus $20 \pm 4 \%, \mathrm{p}=0.02)$ and higher residual volume $(\mathrm{RV}) /$ total lung capacity ratio $(0.69 \pm 0.06$ versus $0.75 \pm 0.05, \mathrm{p}=0.03)$ at baseline, respectively, than those who attended the 2-year follow-up visit $(\mathrm{n}=32)$. We also analysed the baseline Saint George's Respiratory Questionnaire (SGRQ) in patients who did not attend the 2-year follow-up $(\mathrm{n}=11)$ and found a higher score indicating worse quality of life $(70 \pm 10$ versus $58 \pm 12, \mathrm{p}=0.04)$. Changes from baseline at 6 months, 1 year and 2 years are shown in table 1 . The 6 -month and 1-year results were comparable for the overall group and for the group of patients attending the 2-year follow-up visit. At 2 years, both RV and SGRQ improvements remained significant, whereas the 6-min walking test (6MWT), modified Medical Research Council dyspnoea scale, and FEV1 were not statistically different from baseline. While 45 serious adverse events (SAEs) occurred in 26 patients within the first year (0.9 SAEs per patient-year), 27 SAEs in 20 patients occurred within the second year (0.44 SAEs per patient-year). SAEs between the one and 2-year follow-up included 18 respiratory events (12 COPD exacerbation, 4 pneumonia, 1 lung transplant, 1 lung nodule). No pneumothorax, no coil migration, and no unexpected SAEs were observed between one and 2 years of follow-up.

To our best knowledge, this is the first study to report the longer-term safety and efficacy of ECT beyond 1 year from patients randomised to the ECT group in a randomised clinical trial. A strength of this study is the low rate of loss to follow-up with $74 \%$ of evaluable patients returning for the 2 -year visit. We found

@ERSpublications

In severe emphysema, endobronchial coils improve quality of life with an acceptable safety profile at 2 years http://ow.ly/eVlg30gKrPE

Cite this article as: Deslée G, Leroy S, Perotin JM, et al. Two-year follow-up after endobronchial coil treatment in emphysema: results from the REVOLENS study. Eur Respir J 2017; 50: 1701740 [https://doi. org/10.1183/13993003.01740-2017]. 
TABLE 1 Changes from baseline at 6 and 12 months, and 2 years after coil treatment

\begin{tabular}{|c|c|c|c|c|c|c|c|}
\hline & \multicolumn{3}{|c|}{ Overall group } & \multicolumn{4}{|c|}{ Patients who attended the 2-year follow-up visit } \\
\hline & Baseline & 6 months & 12 months & Baseline & 6 months & 12 months & 2 years \\
\hline Subjects $\mathrm{n}$ & 50 & 47 & 44 & 32 & 32 & 32 & 32 \\
\hline$\geqslant 54 \mathrm{~m}$ & & $16 / 44(36 \%)$ & $8 / 43(19 \%)$ & & $12 / 32(38 \%)$ & $7 / 32(22 \%)$ & $7 / 28(25 \%)$ \\
\hline$\geqslant 25 \mathrm{~m}$ & & $19 / 44(43 \%)$ & $12 / 43(28 \%)$ & & $15 / 32(47 \%)$ & $11 / 32(34 \%)$ & $11 / 28(39 \%)$ \\
\hline $\begin{array}{c}\text { mMRC scale } \\
\geqslant 1\end{array}$ & $3 \pm 0.7$ & $\begin{array}{c}-0.5 \pm 0.9 ; p=0.0002 \\
19 / 47(40 \%)\end{array}$ & $\begin{array}{c}-0.5 \pm 1 ; p=0.006 \\
19 / 41(46 \%)\end{array}$ & $2.9 \pm 0.6$ & $\begin{array}{c}-0.7 \pm 0.9 ; p=0.0003 \\
15 / 32(47 \%)\end{array}$ & $\begin{array}{c}-0.7 \pm 1 ; p=0.0007 \\
15 / 30(50 \%)\end{array}$ & $\begin{array}{c}-0.4 \pm 1 ; p=0.06 \\
13 / 30(43 \%)\end{array}$ \\
\hline$\geqslant 100 \mathrm{~mL}$ & & $17 / 47(36 \%)$ & $12 / 44(27 \%)$ & & $12 / 32(38 \%)$ & $8 / 32(25 \%)$ & $8 / 32(25 \%)$ \\
\hline$\geqslant 10 \%$ & & $21 / 47(45 \%)$ & $14 / 44(32 \%)$ & & $14 / 32(44 \%)$ & $9 / 32(28 \%)$ & $12 / 32(38 \%)$ \\
\hline RV L & $6.2 \pm 0.86$ & $\begin{array}{c}-0.53 \pm 0.72 \\
p<0.0001\end{array}$ & $\begin{array}{c}-0.47 \pm 0.68 \\
p<0.0001\end{array}$ & $5.92 \pm 0.74$ & $\begin{array}{c}-0.46 \pm 0.51 \\
p<0.0001\end{array}$ & $-0.39 \pm 0.68 ; p=0.003$ & $\begin{array}{c}-0.28 \pm 0.69 \\
p=0.03\end{array}$ \\
\hline$\geqslant 350 \mathrm{~mL}$ & & $29 / 47(62 \%)$ & $25 / 44$ (57\%) & & $19 / 32$ (59\%) & $18 / 32(56 \%)$ & $14 / 32(44 \%)$ \\
\hline SGRQ $(0-100)$ & $60.8 \pm 12.8$ & $\begin{array}{c}-11.1 \pm 16.1 \\
p<0.0001\end{array}$ & $-9.1 \pm 15.9 ; p=0.0006$ & $57.9 \pm 12.3$ & $\begin{array}{c}-12.3 \pm 17.6 \\
p=0.0005\end{array}$ & $\begin{array}{c}-11.5 \pm 16.4 \\
p=0.0006\end{array}$ & $\begin{array}{c}-7.9 \pm 14.5 \\
p=0.005\end{array}$ \\
\hline
\end{tabular}

Data are presented as mean \pm SD or number $(\%)$, unless otherwise stated. The percentage of patients reaching the minimal clinically important difference for each variable is indicated. Changes from baseline were assessed by paired t-tests. 6MWT: 6-min walking test; mMRC : modified Medical Research Council dyspnoea scale; FEV1: forced expiratory volume 1 s; RV: residual volume; SGRQ: Saint George's Respiratory Questionnaire.

a clinically relevant improvement in coil-treated patients in the REVOLENS study for the SGRQ total score (mean 7.9 points and responder rate 65\%) at 2 years. Despite being statistically significant, the magnitude of change in RV (mean $0.28 \mathrm{~L}$ and responder rate 44\%) might not be clinically relevant. These results are in line with the results obtained in one monocentric study from patients treated by ECT in two early pilot studies and attending voluntary visits at 2 and 3 years [8]. However, our results must be interpreted cautiously owing to limitations in the study. As control patients in our study were offered coil treatment after 1 year, we do not have a control group at 2 years. Outcomes at 2 years after ECT for the control group are not yet available and some patients from the control group were not treated by ECT after 1 year of follow-up because of death, exclusion criteria or patient's refusal. Second, our analyses comparing the 2-year results with baseline data do not take into account the natural clinical and functional decline in severe emphysema, as shown in the control and lung volume surgery groups in the NETT study $[9,10]$. We can therefore not exclude that our results at two years underestimate the effects of ECT compared to the natural history of severe emphysema. However, this is likely balanced by a selection bias presumed to occur in long-term follow-up with the best (healthiest) responders more likely to attend follow-up visits. The patients who died or underwent lung transplantation exhibited lower body mass index and $\mathrm{FEV} 1$, factors known to impact survival in COPD. Although our results are exploratory, they suggest that careful risk assessment is important to set appropriate expectations with patients. The patients who did not attend the 2-year follow-up visit exhibited baseline differences including a higher SGRQ score indicating worst quality of life. A potential criticism of the SGRQ results could suppose a placebo effect especially in the absence of a control group. We do agree that for patients with very severe disease refractory to medical management, enrolment in an interventional trial might engender a placebo effect; however, the highly unlikely scenario of a placebo effect maintained at 1 year becomes implausible at 2 years. Our results do show a large variability of response to treatment across a small population, as shown by large standard deviation for each variable at 2 years. Unfortunately, our sample size is too small to conduct multivariate analyses to propose predictive factors of long-term response to ECT. Nevertheless, a strength of this study is pre-specified investigation of all adverse events between one and 2 years after ECT. No unanticipated events occurred up to 2 years, and SAEs decreased after 1 year. No late pneumothorax or haemoptysis events were reported. This is an important finding, as these complications may lead to life-threatening conditions requiring intervention, such as chest tube placement, surgery or bronchial artery embolisation [11]. Long-term tolerability is crucial as the ECT is considered a permanent treatment, although we described a successful late removal of two coils, 10 months after ECT in one patient initially randomised in the usual care group in the REVOLENS study [12]. We acknowledge that adverse events occurring after 1 year in the patients who did not attend the 2-year follow-up visit may not have been reported despite our best efforts, and that underreporting of adverse events reliant on patient recall may also have occurred. 
In conclusion, this 2-year prospective follow-up study showed that ECT is associated with a sustained improvement in quality of life, sustained decrease in RV and an acceptable safety profile, with no late-onset or unanticipated events occurring in the one to 2-year post-treatment period. Subsequent 5-year follow-up assessments from REVOLENS and other larger long-term clinical trials will help to better define the long-term effectiveness and safety of endobronchial treatments in severe emphysema and will help to solidify its use in the standard of care.

Gaëtan Deslée ${ }^{1}$, Sylvie Leroy ${ }^{2}$, Jeanne Marie Perotin ${ }^{1}$, Hervé Mal $^{3}$, Hervé Dutau, Arnaud Bourdin ${ }^{5}$, Jean Michel Vergnon ${ }^{6}$, Christophe Pison ${ }^{7}$, Romain Kessler ${ }^{8}$, Vincent Jounieaux ${ }^{9}$, Mathieu Salaün ${ }^{10}$, Armelle Marceau $^{3}$, Sandra Dury ${ }^{1}$, Jonathan Benzaquen ${ }^{2}$, Margaux Bonnaire ${ }^{11}$, Sylvain Dukic ${ }^{12}$, Coralie Barbe ${ }^{11}$ and Charles Hugo Marquette ${ }^{2}$, on behalf of the REVOLENS Study Group ${ }^{13}$

${ }^{1}$ Service de Pneumologie, INSERM U903, Hôpital Universitaire, Reims, France. ${ }^{2}$ Service de Pneumologie, CHU de Nice, FHU OncoAge, Université Côte d'Azur, Nice, France. ${ }^{3}$ Service de Pneumologie, Hôpital Universitaire Bichat, Paris, France. ${ }^{4}$ Service d'Oncologie Thoracique, Maladies de la Plèvre, Pneumologie Interventionnelle, Hôpital Universitaire, Marseille, France. ${ }^{5}$ Département de Pneumologie et Addictologie, PhyMedExp, INSERM U1046, CNRS UMR, Hôpital Universitaire, Montpellier, France. ${ }^{6}$ Service de Pneumologie, Hôpital Universitaire, Saint Etienne, France. ${ }^{7}$ Clinique Universitaire de Pneumologie, Pôle Thorax et Vaisseaux, Inserm1055, Hôpital Universitaire Grenoble Alpes, Inserm1055, Université Grenoble Alpes, Grenoble, France. ${ }^{8}$ Service de Pneumologie, Hôpital Universitaire, Strasbourg, France. ${ }^{9}$ Service de Pneumologie, Hôpital Universitaire, Amiens, France. ${ }^{10}$ Service de Pneumologie, Oncologie Thoracique et Soins Intensifs Respiratoires, Hôpital Universitaire, Rouen, France. ${ }^{11}$ Département de Méthodologie, Pôle Recherche-Innovations, Hôpital Universitaire, Reims, France. ${ }^{12}$ Unité de vigilance des essais cliniques, Hôpital Universitaire, Reims, France. ${ }^{13} \mathrm{~A}$ list of members of the REVOLENS Study Group can be found in the acknowledgements section.

Correspondence: Gaëtan Deslée, Service de Pneumologie, INSERM U903, Hôpital Universitaire de Reims, 45, Rue Cognacq-Jay, 51100 Reims, France. E-mail: gdeslee@chu-reims.fr

Received: July 172017 | Accepted after revision: Sept 192017

This study is registered at ClinicalTrials.gov with identifier number NCT01822795.

Support statement: The REVOLENS Study was supported by an academic grant from the French Ministry of Health (Direction Générale de l'Offre de Soins, PSTIC-2012) which required a randomised trial design. The coils were purchased from the manufacturer (PneumRx/BTG, Mountain View, CA, USA) which had no involvement in the study design and analysis of the data. PneumRx/BTG provided a financial support for the follow-up assessment after 1 year. The French Ministry of Health had full access to the results of the study, but had no involvement in the design and conduct of the study, collection, management, analysis, and interpretation of the data, preparation, review, or approval of the manuscript, and decision to submit the manuscript for publication. Funding information for this article has been deposited with the Crossref Funder Registry.

Conflict of interest: Disclosures can be found alongside this article at erj.ersjournals.com

Acknowledgements:

Author's contributions: G. Deslée had full access to all of the data in the study and takes responsibility for the integrity of the data and the accuracy of data analysis. Study concept and design: G. Deslée, C.H. Marquette, C. Barbe; acquisition of data: G. Deslée, S. Leroy, J.M. Perotin, H. Mal, H. Dutau, A. Bourdin, J.M. Vergnon, C. Pison, R. Kessler, V. Jounieaux, M. Salaün, A. Marceau, S. Dury, J. Benzaquen, S. Dukic, M. Bonnaire, C.H. Marquette; analysis and interpretation of data: G. Deslée, C.H. Marquette, C. Barbe; drafting of the manuscript: G. Deslée, C.H. Marquette, S. Leroy, J.M. Perotin; critical revision of the manuscript for important intellectual content: G. Deslée, S. Leroy, J.M. Perotin, H. Mal, H. Dutau, A. Bourdin, J.M. Vergnon, C. Pison, R. Kessler, V. Jounieaux, M. Salaün, A. Marceau, S. Dury, J. Benzaquen, M. Bonnaire, S. Dukic, C. Barbe, C.H. Marquette; statistical analysis: C. Barbe; study supervision: G. Deslée.

Members of the REVOLENS Study Group: The members of the REVOLENS Study Group include all authors and Hervé Vallerand, François Lebargy, Claire Launois (Hôpital Universitaire de Reims), Johanna Pradelli, Matthieu Buscot, Celine Sanfiorenzo, Sylvie Korzeniewski, Andrea Mazzetta, Jennifer Griffonnet, Ariane Guillemart, Demosthenes Makris (Hôpital Universitaire de Nice), Marie Christine Dombret, Frédérique Servin, Marie Pierre Debray (Hôpital Universitaire Bichat, Paris), Sophie Laroumagne, Fabienne Bregeon, Carine Gomez, Philippe Astoul (Hôpital Universitaire de Marseille), Nathalie Lesavre (Centre d'Investigation Clinique 1409, AP-HM, Marseille), Jean Pierre Mallet, Anne Sophie Gamez, Philippe Tarodo, Christophe Brousse, Clément Broissin (Hôpital Universitaire de Montpellier), Yoann Thibout, Fabrice Di Palma, Frédéric Costes (Hôpital Universitaire de Saint Etienne), Amandine Briault, François Arbib, Emilie Reymond (Hôpital Universitaire de Grenoble), Michele Porzio, Benjamin Renaud-Picard (Hôpital Universitaire de Strasbourg), Bénédicte Toublanc, Géraldine François (Hôpital Universitaire d’Amiens), Luc Thiberville, Antoine Cuvelier, Samy Lachkar (Hôpital Universitaire de Rouen), Delphine Gras (Monitoring team, Hôpital Universitaire de Reims), Philippe Benoit (Pharmacie, Hôpital Universitaire de Reims), Annick Leclainche, Damien Jolly (Pôle recherche et innovation, Hôpital Universitaire de Reims). Data and Safety Management Board: François Fourrier, Eric Hachulla, Nicolas Roche, Daniel Dusser. The collaborators did not receive any compensation for their contribution.

\section{References}

1 Slebos DJ, Hartman JE, Klooster K, et al. Bronchoscopic coil treatment for patients with severe emphysema: a meta-analysis. Respiration 2015; 90: 136-145. 
2 Makris D, Leroy S, Pradelli J, et al. Changes in dynamic lung mechanics after lung volume reduction coil treatment of severe emphysema. Thorax 2017; in press [https://doi.org/10.1136/thoraxjnl-2017-210118].

3 Shah PL, Zoumot Z, Singh S, et al. Endobronchial coils for the treatment of severe emphysema with hyperinflation (RESET): a randomised controlled trial. Lancet Respir Med 2013; 1: 233-240.

4 Zoumot Z, Kemp SV, Singh S, et al. Endobronchial coils for severe emphysema are effective up to 12 months following treatment: medium term and cross-over results from a randomised controlled trial. PLoS One 2015; 10: e0122656.

5 Deslée G, Mal H, Dutau H, et al. Lung volume reduction coil treatment vs usual care in patients with severe emphysema: the REVOLENS randomized clinical trial. JAMA 2016; 315: 175-184.

6 Sciurba FC, Criner GJ, Strange C, et al. Effect of endobronchial coils vs usual care on exercise tolerance in patients with severe emphysema: the RENEW randomized clinical trial. JAMA 2016; 315: 2178-2189.

7 Deslee G, Barbe C, Bourdin A, et al. [Cost-effectiveness of lung volume reduction coil treatment in emphysema. STIC REVOLENS]. Rev Mal Respir 2012; 29: 1157-1164.

8 Hartman JE, Klooster K, Gortzak K, et al. Long-term follow-up after bronchoscopic lung volume reduction treatment with coils in patients with severe emphysema. Respirology 2015; 20: 319-326.

9 Naunheim KS, Wood DE, Mohsenifar Z, et al. Long-term follow-up of patients receiving lung-volume-reduction surgery versus medical therapy for severe emphysema by the National Emphysema Treatment Trial Research Group. Ann Thorac Surg 2006; 82: 431-443.

10 Criner GJ, Cordova F, Sternberg AL, et al. The National Emphysema Treatment Trial (NETT) Part II: Lessons learned about lung volume reduction surgery. Am J Respir Crit Care Med 2011; 184: 881-893.

11 Simon M, Ittrich H, Harbaum L, et al. Bleeding complications after endoscopic lung volume reduction coil treatment: a retrospective observational study. Arch Bronconeumol 2016; 52: 590-595.

12 Dutau H, Bourru D, Guinde J, et al. Successful late removal of endobronchial coils. Chest 2016; 150: e143-e145. 